\title{
Cavitating pulmonary cryptococcosis developing in an HIV antibody patient despite prior treatment with fluconazole
}

\author{
R J Coker, D Bell, B S Peters, S M Murphy
}

\begin{abstract}
Disseminated cryptococcosis developed in an HIV antibody positive patient who was taking fluconazole for oral candidiasis. This case highlights the poor response to therapy that may be seen, and the severe pulmonary complications that may ensue. The use of fluconazole prior to the development of cryptococcosis did not confer protection.
\end{abstract}

\section{Introduction}

Cryptococcus neoformans is a rare but significant cause of pneumonia in patients infected with the human immunodeficiency virus (HIV). The spectrum of disease caused by the fungus varies considerably from asymptomatic pulmonary cryptococcosis to widespread disseminated disease including, commonly, meningitis. ${ }^{12}$ The drug of choice in the treatment of this disease is amphotericin $B$, but recently interest has focused on the role of the azoles, particularly fluconazole.

\section{Case report}

Four weeks prior to admission with headache, a 37 year old West Indian heterosexual was found to be HIV antibody positive after he presented with oral candidiasis. Serum cryptococcal antigen at this time was negative. He was started on fluconazole ( $50 \mathrm{mg} /$ day).

On examination, at admission, he was pyrexial $\left(38^{\circ} \mathrm{C}\right)$ with mild neck stiffness. A chest radiograph revealed soft shadowing in the left upper zone. Examination of the cerebrospinal fluid (CSF) showed budding yeast on India ink stain which was subsequently cultured and identified as Cryptococcus neoformans var. neoformans. Blood cultures and sputum cultures also grew $C$ neoformans. The MIC to fluconazole of $C$ neoformans isolated from the CSF sample was $25 \mathrm{mg} / \mathrm{ml}$. He was hyponatraemic.

He was treated with amphotericin B $(0.6 \mathrm{mg} / \mathrm{kg} /$ day $)$ for three weeks but developed renal impairment (creatinine clearance falling to $28 \mathrm{ml} /$ minute) and subsequently fluconazole (400 mg/day), and flucytosine $(100 \mathrm{mg} / \mathrm{kg} /$ day $)$ but he deteriorated and developed leucopenia (WBC count $\left.0.7 \times 10^{9} / 1\right)$. Further chest radiographs (fig 1 ) and CT (fig 2) showed increasing consolidation with cavitation and scattered intrapulmonary nodules. Bronchoscopic examination with bronchoalveolar lavage confirmed pulmonary cryptococcosis. He was treated for a further month with liposomal amphotericin B ( $3 \mathrm{mg} / \mathrm{kg} /$ day) but continued to deteriorate and died two months after admission.

Postmortem examination revealed widespread involvement in both lungs by $C$ neoformans. No other pathogens were detected.

\section{Discussion}

Cryptococcal infection occurs in $5-10 \%{ }^{13-7}$ of all patients with the acquired immunodeficiency syndrome (AIDS) and is the fourth most common infection found in AIDS patients after Pneumocystis carinii, cytomegalovirus, and mycobacterial infections. ${ }^{8}$ Cryptococcosis is the initial manifestation of AIDS in $40 \%$ to $45 \%$ of such patients.

The lung is the usual site of entry of $C$ neoformans and of those infected $50 \%$ to $80 \%$ are immunocompromised. ${ }^{9}$ Cryptococcal infection may be limited to the lungs in immunocompetent hosts, whereas disseminated disease and meningitis ${ }^{10}$ are more common in immunocompromised hosts. Pulmonary cryptococcosis is probably more common

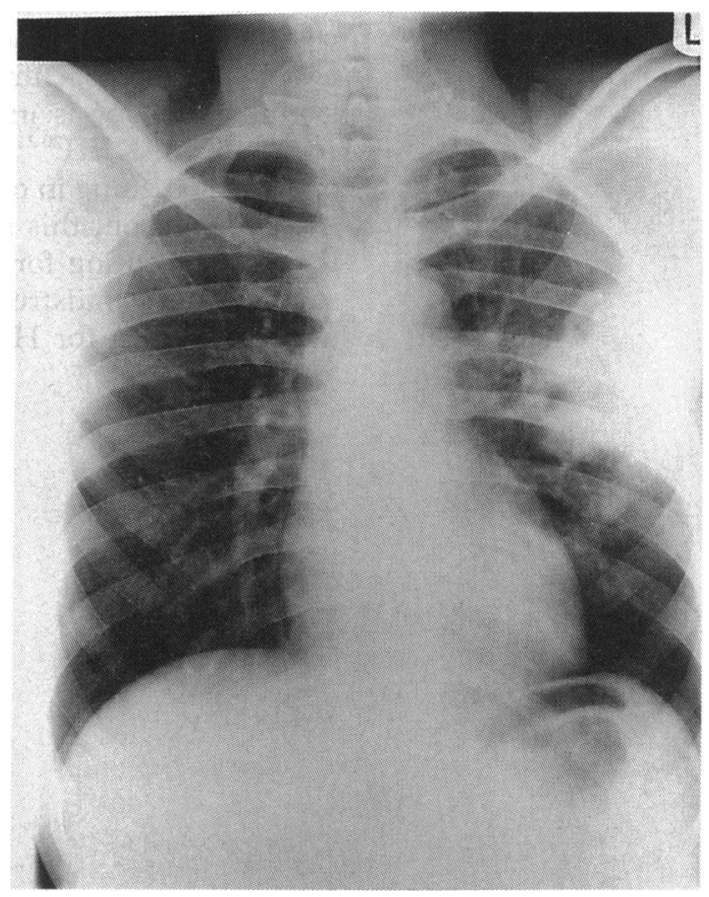

Fig 1 Chest radiograph 6 weeks after admission showing involvement of both lung fields and cavitation in the left lung.
Address correspondence to: Dr Coker

Accepted for publication 22 October 1991 
Fig 2 CT scan of the thorax at 6 weeks showing cavitation in the left lung.

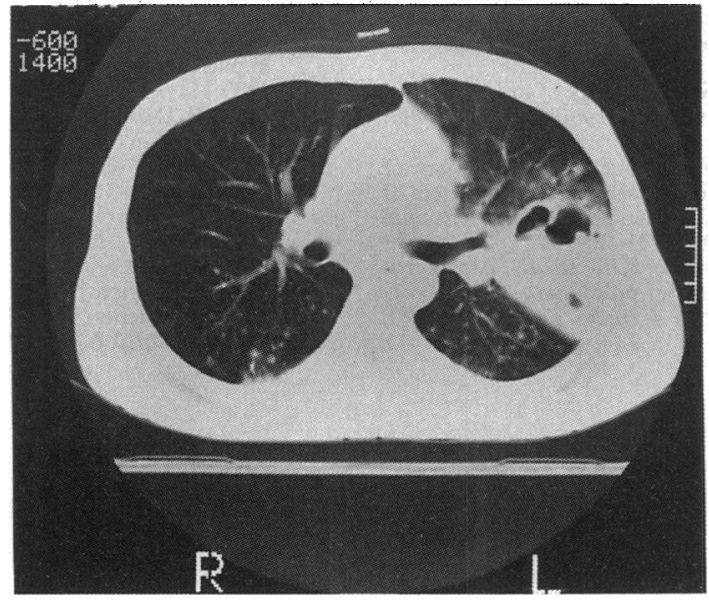

than has been previously realised; Cameron $e t$ $a l^{2}$ reported an incidence of $39 \%$ in AIDS patients with cryptococcosis and Zuger et al found that 6 of $22(27 \%)$ patients who presented with cryptococcal meningitis had pulmonary involvement. ${ }^{3}$ Cavitation is, however, unusual. ${ }^{9112}$ It appears to be commoner in immunocompromised patients. In 15 patients studied by Khoury et $a l^{9}$ who were immunocompromised owing to corticosteroid therapy, malignancy, alcoholism, or post-operative status, the presentation varied widely from solitary or multiple pulmonary nodules, with and without cavitation, to patchy infiltration, pleural effusion, or adenopathy. Of the nine immunocompetent patients studied none developed cavitation and lung involvement was less extensive. However, in contrast, none of the seven AIDS patients studied by Miller et $a l^{13}$ had large nodules, cavitation, or alveolar infiltration and none of the 12 AIDS patients in the review by Cameron et $\mathrm{al}^{2}$ had evidence of cavitation.

$\mathrm{T}$-cell-dependent responses are the primary factors responsible for recovery from cryptococcal infection, ${ }^{14}$ thus explaining the poor outlook in AIDS patients. The inability of the host to mount an inflammatory response necessary to make a nodule or infiltrate radiographically apparent has been suggested in AIDS, in contrast to other immunocompromised patients without AIDS. ${ }^{13}$ The case described here shows the unusual development of widespread nodules and cavitation in the context of AIDS. The more pronounced radiographic features may have resulted from less marked immunosuppression in this case though immunological markers are unfortunately unavailable. Alternatively prior treatment with fluconazole may have altered the natural progression of the disease.

Fluconazole is effective in the treatment of oral candidiasis, and has been used with success in the treatment of cryptococcosis. ${ }^{15}$ It is a selective inhibitor of fungal cytochrome P450 which is necessary for the synthesis of ergosterol, ${ }^{16}$ the essential sterol in fungal membrane. Amphotericin B binds to ergosterol causing increased cell permeability and cell death. Though hyponatraemia and positive cultures for cryptococci from extrameningeal sources, as seen in this patient, indicate a poor prognosis,' prior therapy with fluconazole may have reduced ergosterol levels and therefore have prevented full benefit from amphotericin B. Alternatively, use of fluconazole prior to the development of cryptococcosis may have selected an isolate with a high MIC to fluconazole and poor response to the higher dose subsequently given. However, the laboratory sensitivity of azole antifungal drugs is highly dependent on test conditions, ${ }^{17}$ and MICs for responsive strains that are higher than the concentration of the drug attained in the responsive patient may be seen.

Liposomal amphotericin B (AmBisome) has been used with success in the treatment of cryptococcal meningitis, ${ }^{18}$ pulmonary aspergillosis, ${ }^{19}$ and other systemic fungal infections. ${ }^{20}$ It appears to be less nephrotoxic than conventional amphotericin $B$, hence its use here, though its efficacy is still unknown. Results of clinical trials are awaited. Flucytosine may be effective but is limited by its considerable toxocity profile, ${ }^{162122}$ as was the experience in this case. The current dosage recommendation of $150 \mathrm{mg} / \mathrm{kg} /$ day may be too high and $75-100 \mathrm{mg} / \mathrm{kg} /$ day is perhaps adequate. ${ }^{23}$

Awareness that cryptococcal infections may occur despite patients already taking systemic antifungal drugs is important. Further studies are required to establish possible interactions between the azoles and amphotericin $B$. Though unusual, cavitation may be a feature of pulmonary cryptococcosis in AIDS.

1 Chuck S, Sande M. Infections with Cryptococcus neoformans in the acquired immunodeficiency syndrome. N Engl J Med 1989;321:794-9.

2 Cameron ML, Bartlett JA, Gallis HA, Waskin HA. Manifestations of pulmonary cryptococcosis in patients with acquired immunodeficiency syndrome. Rev Infect Dis 1991;13:64-7.

3 Zuger A, Louie E, Holzman RS, Simberkoff MS, Rahal JJ Cryptococcal disease in patients with the acquired immunodeficiency syndrome. Diagnostic features and outcome of treatment. Ann Intern Med 1986;104:234-40.

4 Eng RHK, Bishburg E, Smith SM, Kapila R. Cryptococcal infections in patients with acquired immune deficiency syndrome. Am J Med 1986;81:19-23.

5 Kovacs JA, Kovacs AA, Polis M, et al. Cryptococcosis in the acquired immunodeficiency syndrome. Ann Intern Med 1985;103:533-8.

6 Dismukes WF. Cryptococcal meningitis in patients with AIDS. J Infect Dis 1988;157:624-7.

7 Weinke T, Rogler G, Sixt C, et al. Cryptococcosis in AIDS patients: observations concerning CNS involvement. $J$ peurol 1989;236:38-42.

8 Diamond RD. Cryptococcus neoformans. In: Mandel GL Douglas RG, Bennett JE, (eds). Principles and Practice of Infectious Diseases. New York: Churchill Livingston 1990:1980-89.

9 Khoury MB, Godwin JD, Ravin CE, Gallis HA, Halvorsen RA, Putman CE. Thoracic cryptococcosis: immunologic competence and radiologic appearance. $A J R$ 1984;141 893-6.

10 Kerkering TM, Duma RJ, Shadomy S. Evolution of pulmonary cryptococcosis. Clinical implications from a study of 41 patients with and without compromising host factors. Ann Intern Med 1981;94:611-6.

11 Warr W, Batts JH, Stone A. The spectrum of pulmonary cryptococcosis. Ann Intern Med 1968;69:1109-16.

12 Feigin DS. Pulmonary cryptococcosis: radiologic-pathologic correlates of its three forms. AJR 1983;141:1263-72.
latic

13 Miller WT Jr, Edelman JM, Miller WT. Cryptococcal puller WT Jr, Edelman JM, Miller WT. Cryptococcal
appearance. Radiology 1990;175:725-8.

14 Miller GP. The immunology of cryptococcal disease. Semin Respir Infect 1986;1:45-52.

15 Sugar AM, Stern JJ, Dupont B. Overview: treatment of cryptococcal meningitis. Rev Infect Dis 1990;12 Suppl 3:S338-48.

16 Van den Bossche H, Willemsens G, Cools W, Lauwers WFJ, 
Le Jeune L. Biochemical effects of miconazole on fungi Inhibition of ergosterol biosynthesis in Candida albicans. Chem Biol Interact 1978;21:59-78.

17 Odds FC. Laboratory tests for the activity of imidazole and trazole antifungal agents in vitro. Semin Dermatol 1985; 4:260-70.

18 Coker $R$, Tomlinson D, Harris J. Successful treatment of cryptococcal meningitis with liposomal amphotericin B after failure of treatment with fluconazole and conventional amphotericin B. AIDS 1991;5:231-2.

19 Katz NM, Pierce PF, Anzeck RA, et al. Liposomal amphotericin B for treatment of pulmonary aspergillosis in a heart transplant patient. $J$ Heart Transplant 1990; in a heart
20 Lopez-Berestein G, Feinstein V, Hopfer R, et al. Liposomal amphotericin $B$ for treatment of systemic fungal infection in patients with cancer: a preliminary study. $J$ Infect Dis 1985;151:704-10.

21 Bennet JE, Dismukes WE, Duma RJ, et al. A comparison of amphotericin $B$ alone and combined with flucytosine in the treatment of cryptococcal meningitis. $N$ Engl J Med 1979;301:126-31.

22 Sugar AM, Stern JJ, Dupont B. Overview: treatment of cryptococcal me

23 Shaunak $S$, Cohen J. Clinical management of fungal infection in patients with AIDS. $J$ Antimicrob Chemother tion in patients with A
$1991 ; 28$ (suppl A):67-81. 